\title{
A study on the incidence of avascular necrosis following closed reduction of the dislocated hip in children with congenital dysplasia of the hip: a suggestion on clinical management
}

\begin{abstract}
Background: Our district general hospital is one of two providers of elective paediatric orthopaedic services to a rural population with an annual live birth rate of approximately 5000 per year. The preferred practice in our hospital for dislocated hips in cases of congenital dysplasia of the hip (CDH) is to place the child in traction for 2 weeks then perform an arthrogram and closed reduction followed by the application of a hip spica. We wanted to evaluate the incidence of avascular necrosis (AVN) of the femoral head following closed reduction of the hip.

Methods: We have retrospectively reviewed all case notes for patients with congenital dysplastic hips $(\mathrm{CDH})$ that had dislocated hips over a 20 year period from 1993-2013. Data was collected from clinic letters and radiographs.

Results: There were 34 children with $\mathrm{CDH}$ and dislocated hips. The mean $( \pm$ S.D) age at closed reduction was $14( \pm 6)$ months. The mean follow up period was to $6( \pm 3)$ years. One patient had features of AVN noted on radiographs at age 7 years $(2.9 \%)$. This patient's dislocated hip was reduced closed at age 8 months. In our series only 3 patients required open reduction. Of these 2 were followed by femoral derotation osteotomies. All these 3 patients presented with dislocated hips at greater than 15 months of age. Clinically all patients were functioning with no restrictions at last follow up

Conclusion: We have treated children with arthrogram and closed reduction of the hip from the age of 4 months to 33 months resulting in one case of AVN in a child treated at 8 months old. This gives an AVN rate of 1 in 34 (2.9\%). We would recommend this to be an acceptable method of management with a very low rate of AVN
\end{abstract}

Volume 5 Issue 5 - 2016

\section{Kohila Vani Sigamoney, Jonathan Kent, Terence Savaridas, Carol Brignall}

Cumberland Infirmary Carlisle, UK

Correspondence: Kohila Vani Sigamoney, Cumberland Infirmary Carlisle, Northern Deanery, UK, Email kohilavani_sigamoney@yahoo.com

Received: July 19, 2016 | Published: September 14, 2016

\section{Introduction}

Congenital dysplasia of the hip refers to alterations in hip growth and stability in utero and in the newborn period. It may result in dysplasia, with a spectrum of problems including dislocation of the hip joint.

Cumberland Infirmary Carlisle is a district general hospital which was one of two providers of elective paediatric orthopaedic services to a rural population with an annual live birth rate of approximately 5000 per year. The preferred practice by a single surgeon here is to place the child in traction for 2 weeks then perform an arthrogram and closed reduction of the hip followed by the application of a hip spica. This was changed every 6 weeks for a period of 6 months in total. When the hip is reduced, the commonest complication is avascular necrosis (AVN) of the femoral head. Race and Herring (1983) reported 57\% incidence of avascular necrosis if the femoral head is not fully reduced. They also reported good results with closed treatment in $62.5 \%$ of patients before the age of 2 years. ${ }^{1}$ Clarke in 2005 looked at 50 hips and the rate of significant AVN (>grade 1 Kalamchi classification) was $7 \%$ in closed reduction and $14 \%$ in open reduction. ${ }^{2}$ We wanted to evaluate our practice in relation to the incidence of $\mathrm{AVN}$ and performed a retrospective study.

\section{Materials and methods}

The study was undertaken retrospectively and all case-notes for patients (paediatric, under 16 years old) with congenital dysplasia of the hip (CDH) over a period of 20 years (1993-2013) were reviewed. We looked at patient age, age at closed reduction, position of spica, duration in spica, and if further surgery was needed. We also looked at age at last follow up, and if there was avascular necrosis and if there was re-dislocation of the hip. Patients were reviewed with radiographs and clinically assessed using the Consultant's clinic letters from the case notes. Here, observer bias was reduced as the data was collected based on the senior author's view.

\section{Results}

We reviewed 34 children who had dislocated hips. The mean $( \pm$ S.D) age at closed reduction was $14( \pm 6)$ months, range 4 to 33 months. The mean follow up age was $6( \pm 3)$ years old, with a range of 2 to 11 years old. The period of follow-up ranged from 7 months to 9 years. All cases were unilateral and we did not see any children with hip dislocations below the age of 4 months in our series.

The commonest position in the hip spica was 90 degrees of flexion, 45 degrees of abduction about 25 degrees of internal rotation depending on the most congruous position during the arthrogram.

One patient had features of AVN (type 1) noted on radiographs at age 7 years $(2.9 \%)$. This patient's dislocated hip was reduced closed at age 8 months. In our series only 3 patients required open reduction which was followed by femoral derotation osteotomies 6 weeks later in 2 patients. The other had a re-dislocation following initial closed reduction and required an open reduction and application of hip spica. 
All these 3 patients were noted to have dislocated hips at 15 months of age at presentation. However, many patients who were older had successful closed reductions and therefore we cannot conclude in this study that a higher age meant closed reduction was unlikely to work. Clinically all patients were functioning with no restrictions at their last follow up.

\section{Discussion}

In 2013, Kaneko et al reported an AVN rate of $2.7 \%$ using gradual reduction with overhead traction and suggested this method to reduce AVN for patients over 6 months old and leads to lower rates of avascular necrosis. ${ }^{3}$ Below the age of 6 months, bracing is suggested to be the first line treatment. ${ }^{4}$ Despite our mean age of closed reduction being greater than 6 months we have seen a very low rate of AVN (2.9\%). Another study which was a survey done looking at the treatment strategies in France, as practiced by French Society of Paediatric Orthopaedics (SOFOP) members, suggest that AVN rates with progressive closed reduction compared favourably with surgical reduction in the long term for patients treated under the age of $3 .^{5}$

Although ours is a small study, it suggest that using traction followed by arthrogram and closed reduction and application of a hip spica for 6 months in total with 6 weekly changes is a good method of treatment with a low complication rate.

To date, there are no nationally agreed guidelines on the screening and management of dislocated hips in $\mathrm{CDH}$. We would hope that our suggested method of treatment could be considered an acceptable method of conservative treatment.

\section{Conclusion}

We have treated children with arthrogram and closed reduction of the hip from the age of 4 months to 33 months resulting in one case of
AVN in a child treated at 8 months old. This gives an AVN rate of 1 in $34(2.9 \%)$. We would recommend this to be an acceptable method of management with a very low rate of AVN

\section{Acknowledgments}

None.

\section{Conflicts of interest}

None.

\section{References}

1. Race C, Herring JA. congenital dislocation of the hip: an evaluation of closed reduction. J Pediatr Orthop. 1983;3(2):166-172.

2. Clarke NM, Jowett AJ, Parker L. The surgical treatment of established congenital dislocation of the hip: results of surgery after planned delayed intervention following the appearance of the capital femoral ossific nucleus. J Pediatr Orthop. 2005;25(4):434-439.

3. Kaneko H, Kitoh H, Mishima K, et al. Long-term outcome of gradual reduction using overhead traction for developmental dysplasia of the hip over 6 months of age. J Pediatr Orthop. 2013;33(6):628-634.

4. Storer SK, Skaggs DL. Developmental dysplasia of the hip. Am Fam Physician. 2006;74(8):1310-1316.

5. Morin C, Wicart P. Congenital dislocation of the hip, with late diagnosis after 1 year of age: Update and management. Orthop Traumatol Surg Res. 2012;98(6):S154-S158. 